\title{
Encouraging Debut of Immunotherapy In Myeloma
}

\author{
Nahla A M Hamed* \\ Department of Hematology, Alexandria University, Egypt
}

Submission: October 14, 2016; Published: October 21, 2016

*Corresponding author: Nahla A M Hamed, Professor of Hematology, Department of Hematology, Alexandria University, Egypt

\begin{abstract}
Multiple myeloma (MM) is a malignant disorder of antibody-producing clonal plasma cells. It is the second most common hematologic neoplasia worldwide [1]. Despite recent advances in myeloma treatment (high-dose chemotherapy followed by autologous stem cell transplantation, novel immunomodulatory drugs, and proteasome inhibitors); MM remains largely incurable with chemotherapy [2]. This is mostly due to the persistence of minimal residual disease (MRD), which leads to high relapse rates [2]. Furthermore, the prognosis of MM patients who become refractory to recently developed novel agents is very poor [1].

Abbreviations: MM: Multiple Myeloma; MRD: Minimal Residual Disease; PD-L1: Programmed Cell Death Ligand; MDSCs: Myeloid-Derived Suppressor Cells; ACT: Adoptive Cellular Therapy; SLAMF7: Signaling Lymphocyte Activating-Molecule Family Member 7; ADCC: AntibodyDependent Cellular Cytotoxicity; RRMM: Relapsed / Refractory Myeloma; DC: Dendritic Cells; NK: Natural Killer; GM-CSF: Granulocyte- Monocyte Colony-Stimulating factor; C/T: Cancer Testis Antigens; IMiDs: Immunomodulatory Drug; ACT: Adoptive T Cell Therapy; CAR: Chimeric Antigen Receptors; BCMA: B-Cell Maturation Antigen
\end{abstract}

\section{Introduction}

Myeloma is associated with profound immune dysfunction affecting the innate and adaptive immune system. Myeloma cells play also an important role in maintaining immunosuppression [3]. In MM, there is reduced expression of HLA molecules, reduced tumor antigen peptides expression, enhanced expression of inhibitory ligands such as programmed cell death ligand 1(PDL1) and PD-L2, and recruitment of counter-regulatory cells such as $\mathrm{T}$ regulatory cells (Tregs) and myeloid-derived suppressor cells (MDSCs) [4].

The importance of host antitumor immunity is evidenced by long-term molecular CR observed post allogeneic stem cell transplantation [5] due to a graft-versus-myeloma effect exerted by donor-derived T lymphocytes [6]. An overdue era of immune therapy in MM has begun [7].

So far, the only established immuno therapeutic approach for MM is allogeneic stem cell transplantation. However, it is associated with high morbidity and mortality and remains an option for only a fraction of patients [2].

MM immunotherapy can be divided into several categories:

a. Monoclonal antibodies targeting surface molecules present on myeloma cells; b. Monoclonal antibodies targeting checkpoint inhibitors on immune cells;

c. Pharmacologic immunomodulation;

d. Cancer vaccines; and

e. Adoptive cellular therapy (ACT) [3].

\section{Monoclonal antibodies targeting surface molecules present on the myeloma cells}

In MM, several surface molecules have been explored in preclinical and clinical studies as potential targets of monoclonal antibodies. They include SLAMF7 (signaling lymphocyte activating-molecule family member 7), CD38, CD40, CD138, CD56, CD54 (also known as intercellular adhesion molecule 1), IL-6, PD1, CD74, CD162, B2-macroglobulin and GM-2 ganglioside. In 2015, FDA approved daratumumab (anti-CD38) and elotuzumab (anti- SLAMF7) for treatment of myeloma [5].

Monoclonal antibodies targeting CD38: CD38 is a type II transmembrane glycoprotein, expressed at high and uniform levels in most, if not all, plasma cells in all stages of the disease. It is not expressed on primitive hematopoietic precursors (CD34+CD38-), suggesting that hematopoietic recovery would occur following CD38-targeted cytotoxic agents [1]. Both 
Daratumumab and isatuximab are anti-CD38 monoclonal antibodies that trigger antibody-dependent cellular cytotoxicity (ADCC), complement-dependent cytotoxicity and antibodydependent phagocytosis, as well as inhibition of the enzymatic activity of CD38. Moreover, even in the absence of Fc-receptorexpressing effector cells, both monoclonal antibodies can induce direct apoptosis and lysosome-mediated cell death in MM cells harboring p53 mutations. However, CD38 levels alone may not be the sole determinant of sensitivity to daratumumab, because CD38-negative Tregs were also reduced in responsive patients [7].

Daratumumab was approved for use in patients who have failed at least 3 prior lines of therapy [8]. Isatuximab shows single-agent activity in relapsed / refractory myeloma (RRMM) [9]. Daratumumab was administered for prolonged periods at moderate to high doses with little or no toxicity, despite the fact that the CD38 molecule is also expressed at lower levels, on a fraction of hematopoietic cells, cerebellar Purkinje cells, liver and lung smooth muscle cells, and insulin-secreting cells of pancreas [1]. SAR650984 is another anti-CD38 antibody which is under investigation in clinical trials [9].

Monoclonal antibodies targeting SLAMF-7: SLAMF7 is a cell surface glycoprotein receptor highly expressed on MM cells mediating family member 7 adhesion to BM stromal cells. It is selectively expressed on plasma and natural killer (NK) cells and lacks expression on other tissues. Elotuzumab is a fully humanized monoclonal antibody directed against SLAMF-7. It triggers ADCC and enhances NK cell function against MMCs [3]. Elotuzumab was approved in combination with revlimid and dexamethasone for treatment of myeloma who received 1-3 prior therapies [8].

Compared with chemotherapeutic agents, monoclonal antibody therapies appear to be associated with fewer side effects and have distinct mechanisms of action that make them more suitable and effective when combined with other antimyeloma therapies, especially in patients with chemotherapyresistant disease [10].

Monoclonal antibodies targeting checkpoint inhibitors on immune cells: The engagement of $\mathrm{T}$ cell with its target is regulated by a complex balance of costimulatory and coinhibitory bidirectional signals (checkpoints) [3] which allow the cells to turn on and off as needed. Checkpoint inhibitors take the brakes off the immune system [8]. Anti-PD-1 is a checkpoint inhibitor that blocks the inhibitory interaction of PD-1 on T or NK cells with its ligand PD-L1 on tumor cells or tumor-promoting accessory cells [3]. Blockade of PD-1/PD-L1 inhibits accessory cell (plasmacytoid dendritic cells, pDC or MDSCs)-induced MM proliferation and survival while triggering host T- and NK-cell anti-MM cytotoxicity [5].

Recently, the anti-PD-1, pembrolizumab, in combination with Rd (PRd), showed 38\% response rate in lenalidomide refractory patients with an acceptable toxicity profile. The overall response rate was $50 \%$ in a phase $1 / 2$ study combining pembrolizumab with pomalidomide in patients with a median number of 3 prior therapies. Seventy-five percent of patients were double refractory to IMIDs and PIs [3]. These studies demonstrate a role for checkpoint inhibition in MM treatment paradigm and underscore the need for IMIDs immunomodulation to achieve this response [3].

\section{Cancer Vaccines}

Cancer vaccine aims to increase the precursor frequency of antigen-specific $\mathrm{T}$ cells or antibodies through in vivo priming [3]. Vaccination against cancer-specific antigens represents a promising strategy to modulate patient antitumor immune response, particularly in the settings of early-stage or minimal residual disease [5]. Vaccines, in combination with immunomodulatory agents, may serve to achieve and/ or maintain MRD hoping to prolong PFS (and possibly OS) [3]. Several vaccination approaches have been used for myeloma: idiotype vaccines, GM-CSF-based cellular vaccines and dendritic cells (DC)-based vaccines [3].

\section{Idiotype Vaccines}

Idiotype refers to the unique immunological properties of any individual immunoglobulin [6]. The Id is expressed and presented in an HLA-restricted manner on the surface of malignant plasma cells [3]. The secreted idiotype was up taken and presented by dendritic cells and may act as T cell antigens [6]. However, idiotype vaccines were weakly immunogenic and failed to elicit a measurable clinical benefit even when combined with strong adjuvants such as granulocyte-monocyte colonystimulating factor (GM-CSF), IL-12, alum or keyhole limpet hemocyanin [3].

\section{GM-CSF-based cellular vaccines}

GM-CSF-based vaccine consists of 2 allogeneic cell lines: H929 and U266, coupled to a GM-CSF-secreting by stander cell line, K562/GM. This vaccine formulation has been tested in patients with a near complete remission i.e. patients having absent an M-spike and persistence of detectable immuno fixation for 6 months. This study suggested that the generation of tumorspecific immunity in a low disease burden state can significantly delay relapse [3]

\section{DC-based vaccines}

Idiotype-loaded DCs have been used as vaccines in MM patients, mostly in the setting of clinical remission after auto transplant [6]. Decreased regulatory-T cell function and minimal disease state post transplant suggest that this is an optimal setting for vaccination [5]. Cancer testis antigens (C/T) are physiologically expressed in testis and placenta trophoblast only and have a potential role in cell cycle and apoptosis. C/T antigens are up regulated in MM [6]. C/T antigens are considered ideal antigens to target and are the best studied examples 
within this category. They fulfill several parameters, including their low expression on normal tissues and their expression in more aggressive disease and in advanced stage disease [3]. However; their manipulation requires profiling of the $\mathrm{C} / \mathrm{T}$ antigen expression pattern in every MM individual patient [3]. An alternative approach is fusion of DCs with patient-derived tumor cell lysates or apoptotic tumor cells. It optimizes antigen presentation and immune priming against the entire antigenic repertoire of each unique patients' tumor. Presentation of antigens from myeloma cell lines by dendritic cells is greatly enhanced by coating myeloma cells with a specific antibody such as anti-CD138 or use of myeloma cells lysed by repetitive freezethaw cycles [6].

\section{Immunomodulatory drug (IMiDs)}

IMiDs (thalidomide, lenalidomide and pomalidomide) are incorporated into therapies for relapsed / refractory myeloma (RRMM) and newly diagnosed MM. Moreover, maintenance therapy with lenalidomide in both transplant-eligible and -ineligible patients has markedly improved patient outcome [5]. They targets the tumor microenvironment through caspase-8mediated apoptosis; abrogation of MMC binding to BM stromal cells; modulation of cytokine secretion; up regulation of $\mathrm{T}$, $\mathrm{NK}$, and NK-Tcells; and down regulation of $\mathrm{T}$ regulatory cells. Recently, the E3 ubiquitin ligase cereblon has been identified as the molecular target of lenalidomide. Predicated on their immuno stimulatory effects, IMiDs may enhance activities of monoclonal antibodies, checkpoint inhibitors, and vaccines [5].

\section{Adoptive T cell therapy (ACT)}

ACT aims to enhance T-cell antitumor activity through ex vivo manipulations [3]. Myeloma is an ideal disease to treat with adoptive T cell therapy [11]. This can be achieved through T cells engineered to express large numbers of affinity-enhanced TCRs, activated marrow infiltrating $\mathrm{T}$ cells and more recently with cytotoxic T cells endowed with tumor-reactive chimeric antigen receptors (CAR). Among these strategies, CAR-based therapies are perhaps the most appealing, as CART cells recognize their target antigens in an MHC-independent manner [1].

a) CAR T-cell therapy is the adoptive transfer of cytotoxic $\mathrm{T}$ cells that are genetically engineered to express CAR. CAR expressed on the cell surface redirect the cytotoxic $\mathrm{T}$ cells toward the original target of the antibody in a nonHLA-restricted manner [11]. CAR T cells provide a new and currently uncovered spectrum of effector mechanisms against myeloma [12]. There is a fully loaded pipeline of novel CARs targeting myeloma antigens, including CD38 [1] and CD44v6 as well as SLAMF7-CARs [12,13].

b) CD38-CART cells are powerful immunotherapeutic tools since they appeared capable in vitro of eliminating primary CD38+ MM cells from patients who had become resistant to various chemotherapies. This suggests that
CD38-CAR therapy could be a viable choice for patients with few or no further chemotherapy options. Further studies are warranted to diminish the undesired effects of CD38-CART cells against normal CD38+ cells [1].

\section{C) B-cell maturation antigen (BCMA)-CART cells}

BCMA is a target for immunotherapy because it is uniformly expressed on myeloma cells. BCMA has no known expression on normal solid tissue and only very restricted expression on normal hematopoietic cells (normal plasma cells, some memory B cells, and pDC). The observed toxicity was cytokine release syndrome with fever, hypotension and hematologic toxicity. The latter was quite significant in some patients, but within the range that can be explained by the lymphodepleting conditioning regimen and inflammation mediated by BCMA-CART cells in the bone marrow. B-cell depletion is not anticipated when targeting BCMA, as the majority of normal B cells are BCMA negative. The persistence of BCMA-CAR T cells after administration to patients in this trial was relatively short (3 months) [12].

The opportunity for using CAR is for dramatic tumor cell reduction, even in high-risk, refractory MM [5]. It can be used also in the setting of fulminant relapsed disease when there is a need for a rapid reduction in disease burden that would justify the associated toxicity [3]. Moreover, use of lenalidomide and/ or checkpoint inhibitors post-CAR-T cell therapy may allow for persistence of cancer immune surveillance by avoiding T-cell exhaustion [5]. Its efficacy could be improved if combined with immune checkpoint inhibitors and/or with survivin and/or MCL-1 inhibitors which are effective modifiers of cell adhesion-mediated immune resistance induced by the tumor microenvironment [1].

\section{Conclusion}

It is likely that the most effective immunotherapeutic approach for MM will include strategies for expanding the repertoire, function, and persistence of myeloma-directed $\mathrm{T}$ cells as well as enhancing the sensitivity of MM cells to immune attack [4].

\section{References}

1. Drent E, Groen RW, Noort WA, Themeli M, Lammerts van Bueren JJ, et al. (2016) Pre-clinical evaluation of CD38 chimeric antigen receptor engineered $\mathrm{T}$ cells for the treatment of multiple myeloma. Haematologica 101(5): 616-625.

2. Walz S, Stickel JS, Kowalewski DJ, Schuster H, Weisel K, et al. (2015) The antigenic landscape of multiple myeloma: mass spectrometry (re) defines targets for T-cell-based immunotherapy. Blood 126(10): 12031213.

3. Hoyos V, Borrello I (2016) The immunotherapy era of myeloma: monoclonal antibodies, vaccines, and adoptive T-cell therapies. Blood 128(13): 1679-1687.

4. Rapoport AP (2015) Myeloma escape from immunity: an "inside" job. Blood 126 (12): 1401-1402.

5. Bianchi G, Richardson PG, Anderson KC (2015) Promising therapies in multiple myeloma. Blood 126(3): 300-311. 


\section{Cancer Therapy \& Oncology International Journal}

6. Houet L, Veelken H (2006) Active immunotherapy of multiple myeloma European Journal of Cancer 42(11): 1653-1660.

7. Yu-Tzu Tai, Kenneth C Anderson (2016) A new era of immune therapy in multiple myeloma. Blood 128 (3): 318-319.

8. Immuno therapies and antibodies. www://thremmrf.org/multiplemyeloma-knowledge-centrer/myeloma-drugs-guide/.

9. Sonneveld P, Broijl A (2016) Treatment of relapsed and refractory multiple myeloma. Haematologica 101(4): 396-406.

10. Rasche L, Duell J, Castro IC, Dubljevic V, Chatterjee M, et al. (2015) GRP78-directed immunotherapy in relapsed or refractory multiple myeloma - results from a phase 1 trial with the monoclonal immunoglobulin M antibody PAT-SM6. Haematologica 100(3): 377384.

11. Davila ML, Bouhassira DCG, Park JH, Curran KJ, Smith EL, et al (2014) Chimeric antigen receptors for the adoptive T cell therapy of hematologic malignancies. Int J Hematol 99(4): 361-371.

12. Hudecek M, Einsele H (2016) Myeloma CARs are rolling into the clinical arena. Blood 128(13): 1667-1669.

13. Casucci M, Nicolis di Robilant B, Falcone L, et al. (2013) CD44v6targeted $\mathrm{T}$ cells mediate potent antitumor effects against acute myeloid 